\title{
Blind source separation based on JADE algorithm and application
}

\author{
Kangrong Zhang ${ }^{1, ~ a ~, ~ G u a n y u ~} \operatorname{Tian}^{2, \mathrm{~b}}$, Lan Tian ${ }^{1, c^{*}}$ \\ ${ }^{1}$ School of Information Science and Engineering, Shandong University, Jinan, 250100, China \\ ${ }^{2}$ School of Electrical Engineering, Shandong University, Jinan, 250061, China \\ akkrsdu@163.com, ${ }^{\mathrm{b}}$ tian_guanyu@sina.com, \\ * corresponding author: 'tianlan65@sdu.edu.cn
}

Keywords: blind source separation; sound detection; joint approximate diagonalization.

Abstract: Fault monitoring or detection online is very important for the safe operation of power equipment. The traditional contact-type detection system based on vibration feature exist some troubles under the high voltage and strong electromagnetic field conditions. In this paper, a non-contact sound detection method is proposed, which is based on audio features blind source separation (BSS). The joint approximate diagonalization of eigenmatrices (JADE) algorithm is applied in the detection method, which makes use of the forth order statics to automatically suppress Guassian background noises and enhance the non-Gaussian source signals. Compared with the fast independent component analysis (Fast ICA) algorithm, a clearer source signal can be estimated and separated. The simulation experiment results show that by the JADE algorithm, the similar coefficients between the separated signals and the original source signals are all above 0.9, far greater than that by Fast ICA algorithm. So it concludes that the sound BSS method can effectively separate source vibration sound of power equipment and JADE is more efficient than Fast ICA in the fault diagnosis for power equipment.

\section{Introduction}

Power equipment failure will not only cause damage to the device itself, but also cause serious damages to the security, stability and economic operation of the entire power system [1]. Therefore, it is of great importance to detect timely whether the electrical equipment has malfunctioned. For contact fault detection on power equipment, touch sensors are applied. The problem is that these sensors may be interfered or damaged by complex operation environment with high voltage and strong electromagnetic field, which would affect the normal operation of the detection system [2,3]. In addition, the touch sensor, which is very inconvenient to install and maintain, destroy the integrity of external electrical equipment and may bring unexpected serious consequences when failure occurs[4] . Therefore, it is worthy of study and discussion on fault detection of non-contact system.

Experienced engineers can recognize the working state by the sound of power equipment. Thus we can collect audio signals of power equipment through non-contact microphone arrays, and introduce audio analysis technology to detect the fault state [5]. Inevitably there are external interference sound from other vibration sources at equipment operation site, in order to separate the working sound from mixed sounds, here we deal with the problem through BSS technology [6,7]. This paper introduces the blind source separation technology based on ICA and JADE, and then analyzes the performance of the two algorithms in the vibration signal separation to verify the JADE is better than Fast ICA.

\section{Blind source separation of electrical equipment operating sound}

\section{ICA algorithm}

Assuming the source signals $s(t)=\left[s_{1}(t), s_{2}(t), \cdots, s_{N}(t)\right]^{T}$ are mutually independent random variables, and the observed mixed signal is expressed as

$$
x(t)=A s(t)
$$

Where $A_{N \times N}$ is unknown invertible mixing matrix, the goal is to find a matrix W to estimate the source 
signal, paper [8] introduces a fast independent component analysis algorithm, the objective function is given by

$$
J_{G}(w)=\left[E\left\{G\left(w^{T} x\right)\right\}-E\{G(v)\}\right]^{2} .
$$

where $v$ is a random Gaussian vector of zero mean and unit variance, $w$ is a weight vector and $G$ can be any function of non-quadratic. Basic iterative formula is hypothesized as

$$
w_{k+1}=w_{k+1}-\sum_{j=1}^{k} w_{k+1}^{T} w_{j} w_{j}
$$

where $C=\left\{x x^{T}\right\}$ can be covariance matrix of the observed data, Eq.3 keep iterating until $w_{k+1}$ convergent.

\section{JADE algorithm}

(1) Assuming the observed signal with zero mean and unit variance, the pre-whitening procedure is shown in Equation (4):

$$
z(t)=Q x(t)
$$

Four signals are taken as: $z_{i}, z_{j}, z_{k}, z_{l}(1 \leq i, j, k, l \leq N)$, then fourth-order cumulant can be given by Equation (5)

$$
\operatorname{cum}\left(z_{i}, z_{j}, z_{k}, z_{l}\right)=E\left[z_{i} z_{j} z_{k} z_{l}\right]-E\left[z_{i} z_{j}\right] E\left[z_{k} z_{l}\right]-E\left[z_{i} z_{k}\right] E\left[z_{j} z_{l}\right]-E\left[z_{i} z_{l}\right] E\left[z_{j} z_{k}\right]
$$

Then we can sequentially obtain all of the fourth-order cumulants, which can be written as matrix $m=\left[m_{1}, m_{2}, \cdots, m_{N^{2}}\right]$. Then the (i,j) th element of matrix $m$ can be given by Equation (6):

$$
\left[C_{z}(M)\right]_{i j}=\sum_{k, l=1}^{N} \operatorname{cum}\left(z_{i}, z_{j}, z_{k}, z_{l}\right) m_{l k}
$$

Where $\mathrm{M}$ is $n \times n$ arbitrary matrix and $m_{l k}$ is its $(1, \mathrm{k})$ th element. As a feature matrix, the decomposition of cumulant matrix should be satisfied to Equation(7).

$C_{z}\left(M_{i}\right)=\lambda M_{i}$

where $\lambda$ is the eigenvalue.

(2) Then $\mathrm{M}$ is processed by joint approximate diagonalization to determine the unitary matrix $\mathrm{V}$, which is used to obtain the estimation of source signals. The diagonalization process is expressed as:

$$
C_{z}\left(M_{i}\right)=V^{T} C\left(M_{i}\right) V=\operatorname{Diag}\left[k_{4}\left(s_{1}\right), k_{4}\left(s_{2}\right), \cdots, k_{4}\left(s_{N}\right)\right]
$$

Finally we need to make each matrix to be approximately diagonalized, and the basic criteria is to consider off-diagonal elements when it comes close to zero [9], so we have

$$
\min C(U)=\sum_{M_{i} \in M} \text { off }\left[U^{T} C(M) U\right]
$$

Where $\operatorname{off}(\cdot)$ is defined as $\operatorname{off}\left(M_{i}\right)=\sum_{i \neq j}\left|m_{i j}\right|^{2}$, then W is supposed as

$$
W=U^{T} Q
$$

\section{Valuation of separation performance}

The similarity coefficient is commonly used to test the effect of blind source separation algorithm, supposing that $s_{i}(t)$ is the ith source signal of $s(t), y_{j}(t)$ is the corresponding separated signal after BSS, so the similarity coefficient can be given by:

$$
p_{i j}=\frac{\operatorname{cov}\left(s_{i}(t), y_{j}(t)\right)}{\sqrt{\operatorname{cov}\left(s_{i}(t), s_{i}(t)\right) \operatorname{cov}\left(y_{j}(t), y_{j}(t)\right)}}
$$

Where $\operatorname{cov}(\cdot)$ represents variance and the range of $p_{i j}$ is $0 \leq p \leq 1$. 


\section{Simulation and analysis}

We use a microphone array to collect the normal working sound signals at transformer spot [10], denoted as s1. Similarly we obtain wind sound s2, birds singing s3 and human voice s4. The waveforms of the four sources are plotted in Fig.1.

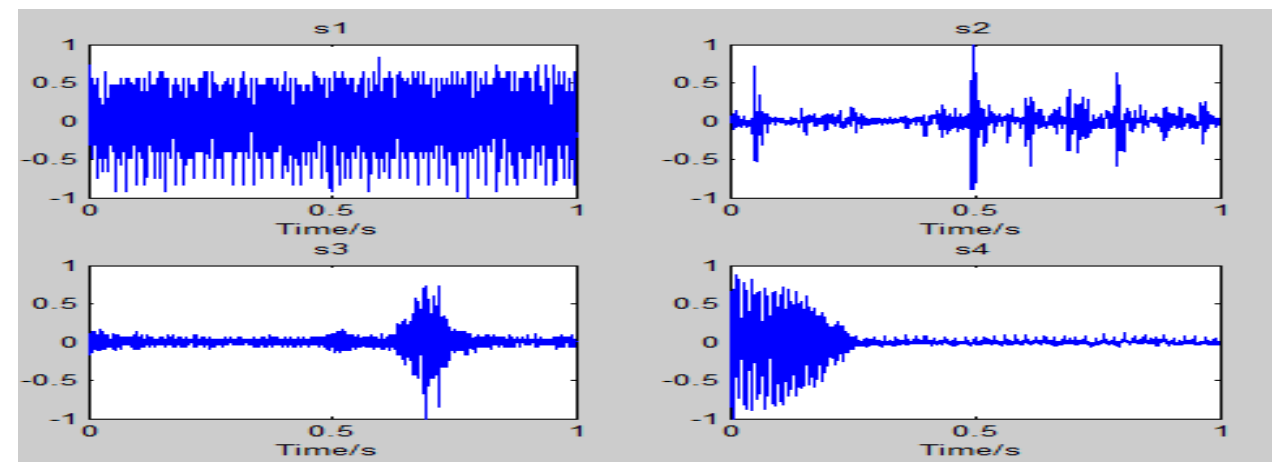

Fig.1 Time waveforms of the four source signals

Firstly, we use Fast ICA to separate the mixed-signals from the four sources. The separated signals waveforms are shown in Fig.2. The results demonstrate that the separated signals have restored the source signal in some extent, but it is not clear enough, so the Fast ICA algorithm may cause error when determine the state of electrical equipment.
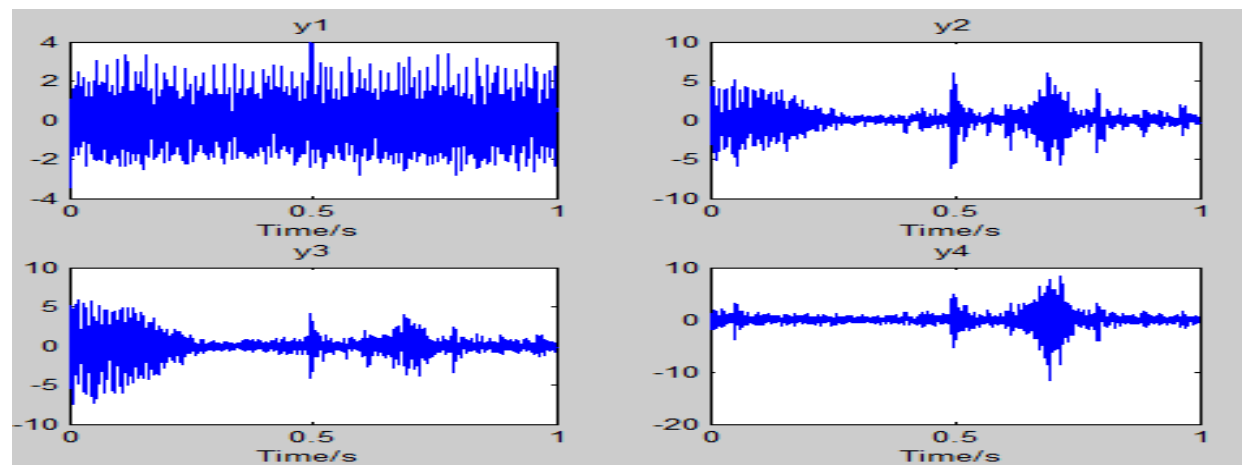

Fig.2 Time waveforms of the separated signals based on Fast ICA

And then, we use JADE algorithm to separate the mixed-signals. The separated waveforms are plotted in Fig.3. We can find the waveforms are substantially the same as the source signal respectively.
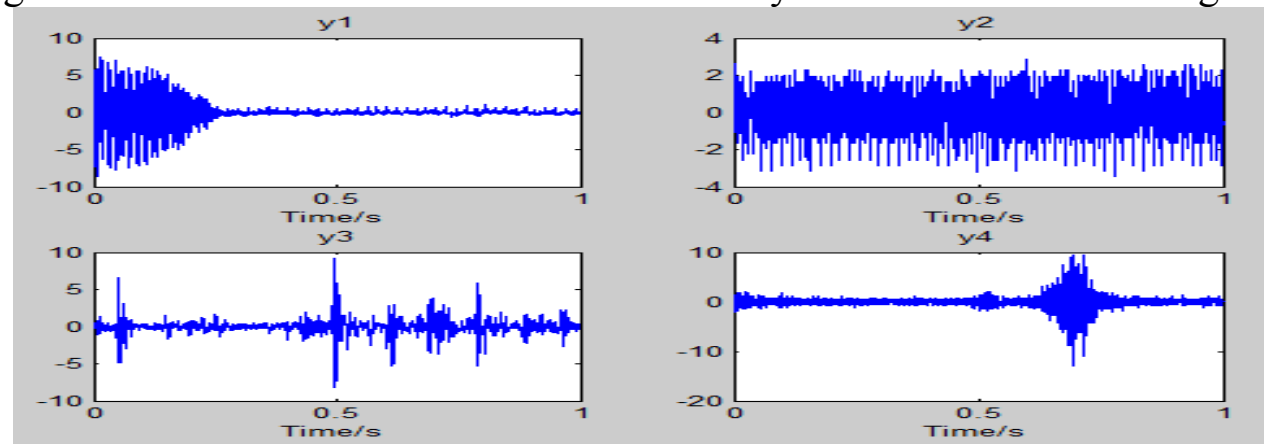

Fig.3 Time waveforms of the separated signals based on JADE

We have done the simulation for five times, and respectively obtain the similarity coefficient matrixs, $M_{1}$ and $M_{2}$. 


$$
M_{1}=\left(\begin{array}{llll}
0.9456 & 0.6379 & 0.7894 & 0.7899 \\
0.9714 & 0.7083 & 0.8004 & 0.5591 \\
0.9386 & 0.7916 & 0.8773 & 0.9045 \\
0.9714 & 0.6781 & 0.8319 & 0.8054 \\
0.9658 & 0.7083 & 0.8004 & 0.5797
\end{array}\right), M_{2}=\left(\begin{array}{cccc}
0.9997 & 0.9998 & 0.9998 & 1 \\
0.9999 & 0.9999 & 1 & 0.9999 \\
0.9967 & 0.956 & 0.9995 & 0.9938 \\
0.9999 & 0.9999 & 1 & 0.9996 \\
0.9998 & 0.9999 & 0.9989 & 0.9999
\end{array}\right) .
$$

Where $M_{1}$ is the similarity coefficient of Fast ICA and $M_{2}$ is the similarity coefficient of JADE .It shows that the similarity coefficient of JADE substantially maintained at 0.9 or greater, achieving better separated results than Fast ICA, it shows that the JADE is more suitable to separate source signal than Fast ICA under this application.

\section{Conclusion}

Due to the transformer sound, human voices, wind sound and birds singing are produced by different vibration sources, which are statistically independent from each other, so this paper applies blind source separation technique into the on-line monitoring sound of electrical equipment, and JADE algorithm, with characteristics of automatically suppressing Gaussian background noises and enhancing the non-Gaussian source signals, is adopted to separate the mixed source sounds. The experimental results show that JADE algorithm obtained the much higher consistency with source signals and so it can be used to judge the working status of device more accurately.

\section{Acknowledgment}

This work was supported by NSF of Shandong Province (ZR2012FM030) and NSFC (11474185).

\section{References}

[1] ZHAO Zhen bing, YUAN Jin sha, GAO Qiang, LUO Guang xiao. Blind Source Separation and Its Application in Temperature On line Monitoring for Electrical Equipment[J].High Voltage Engineering.2007.33(8):18 19.

[2] WANG Nan, CHEN Zhi ye, LU Fang cheng. A survey of on line monitoring and diagnosis for capacitive equipment [J].Power System Technology.2003.27(8):72 76.

[3] HEN Wei-rong, SONG Yong-hua, SUN Jin-xin. Concept and Present Situation of Condition Monitoring of Power System Equipment [J].Power System Technology.2000.24(11):12-14.

[4] Guo Jun, Ji Shengchang, Shen Qi, Zhu Lingyu, Ou Xiaobo, Du Liming. Blind Source Technology for the Detection of Transformer Fault Based on Vibration Method [J]. Tansactions of China Electrotechnical Society, Oct.2012(10): 69-71.

[5] PAN Liangliang,ZHAO Shutao,LI Baoshu.Electrical equipment fault diagnosis based on acoustic wave signal analysis[J]. Electric Power Automation Equipment.2009.29(8):87 88.

[6] Li shun-ming(2001).Blind Source Separation Technology and Application of Vibration Signal[M]. Beijing, China: Aviation Industry Press.

[7]Li Chang-li. Study on Some Algorithms for Blind Source Separation and Their Applications[D].Xian, China: Xidian University,2010.

[8] Xianxue Fan, Jorge Igual, Raul Llinares, Addisson Salazar and Gang Wu. Blind Signal Separation in Distributed Space-Time Coding Systems Using the FastICA Algorithm [J]. Proceedings of International Joint Conference on Neural Networks, July 31-August 5,pp.2925-2929. IEEE.2011.

[9] Antoine Souloumiac. Nonorthogonal Joint Diagonalization by Combining Givens and Hyperbolic Rotations [J].IEEE Trans .Signal Process,vol 57,no 6,pp.2222-2230,Jun 2009.

[10] Ma Xiao-hong, Liang Li-li, Yin Fu-liang. A Voice Activity Detection Method Based on Blind Source Separation for Microphone Array Signals [J].Journal of Electronics \& Information Technology. Mar.2007.pp5895-90. 